\title{
Transaortic septal myectomy: techniques and pitfalls
}

\author{
Anthony Ralph-Edwards ${ }^{1}$, Rachel D. Vanderlaan ${ }^{1}$, Pietro Bajona ${ }^{2,3}$ \\ ${ }^{1}$ Division of Cardiovascular Surgery, Toronto General Hospital, University of Toronto, Toronto, ON, Canada; ${ }^{2}$ Department of Cardiovascular and \\ Thoracic Surgery, University of Texas Southwestern Medical Center, Dallas, TX, USA; ${ }^{3}$ Institute of Life Sciences, Sant'Anna School of Advanced \\ Studies, Pisa, Italy \\ Correspondence to: Anthony Ralph-Edwards, MD. Division of Cardiovascular Surgery, Toronto General Hospital, University of Toronto, 200 \\ Elizabeth St 4N-448 Toronto, Ontario M5G 2C4, Canada. Email: Anthony.ralph-edwards@uhn.ca.
}

\begin{abstract}
Hypertrophic cardiomyopathy (HCM) is the most common congenital cardiac disease, affecting up to 1 in 200 individuals. When it causes left ventricular outflow tract (LVOT) obstruction, treatment is indicated to reduce symptoms and the risk of sudden cardiac death. Pharmacologic therapy is the first line treatment, however if it fails, surgical myectomy or percutaneous ablation of the hypertrophic myocardium are the standard therapies to eliminate subaortic obstruction. Both surgical myectomy and percutaneous ablation have been demonstrated as safe and effective treatments; however, myectomy is the gold standard with a significantly lower complication rate and more complete and longstanding reduction of LVOT obstruction.
\end{abstract}

Keywords: Hypertrophic obstructive cardiomyopathy; septal myectomy; surgical technique

Submitted Mar 03, 2017. Accepted for publication Apr 04, 2017.

doi: $10.21037 /$ acs.2017.07.08

View this article at: http://dx.doi.org/10.21037/acs.2017.07.08

\section{Introduction}

Surgical correction of left ventricular outflow tract (LVOT) obstruction in symptomatic patients with hypertrophic obstructive cardiomyopathy (HOCM) is considered the gold standard of treatment in patients failing medical management (1). Operative techniques have evolved over the years and various adjunctive procedures have been advocated. In a recent review of consecutive patients receiving operations over a 10 -year period at the Toronto General Hospital, we have found that the vast majority of patients with isolated outflow tract obstruction or mixed outflow tract and mid-cavitary obstruction obtain excellent results with a Morrow type resection including extension under the membranous septum. In dedicated HOCM centres, mortality is typically less than $1 \%$ for isolated myectomies and is associated with excellent long-term survival (2-4).

\section{Preparation}

It is most important to obtain a preoperative trans-thoracic echocardiogram (TTE) and a cardiac MRI scan prior to surgery. These studies provide the best estimation of septal thickness and are critical to planning the extent of the surgical resection. A transesophageal echocardiogram (TEE) is performed intra-operatively; however, estimates of the septal thickness and outflow tract gradients on this study are less reliable and should not replace a preoperative transthoracic echocardiogram. TEE is more useful in demonstrating the length and character of the hypertrophied septum and is invaluable in assessing postoperative results. Preoperative MRI will provide important information regarding the degree of fibrosis and the presence of any abnormalities of the sub-valvular apparatus.

\section{Exposition}

Transaortic septal myectomy is performed via a standard median sternotomy incision. The pericardium is opened vertically over the centre of the ascending aorta. Multiple pericardial sutures are used to suspend the right pericardium to the adjacent superior sternal edge. Left pericardial suspension sutures are placed from the aortic annulus level to the pericardial reflection only as this allows the left ventricular apex to drop into the left chest. This maneuver 


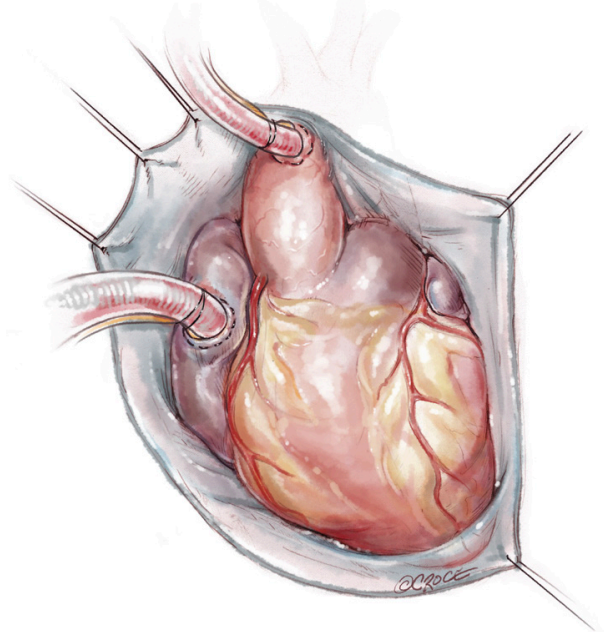

Figure 1 Multiple pericardial sutures are used to suspend the right pericardium to the adjacent superior sternal edge. Left pericardial suspension sutures are placed from the aortic annulus level to the pericardial reflection only, this allows the LV apex to drop into the left chest. This maneuver elevates the aorta to the sternal level and facilitates visualization during the procedure.

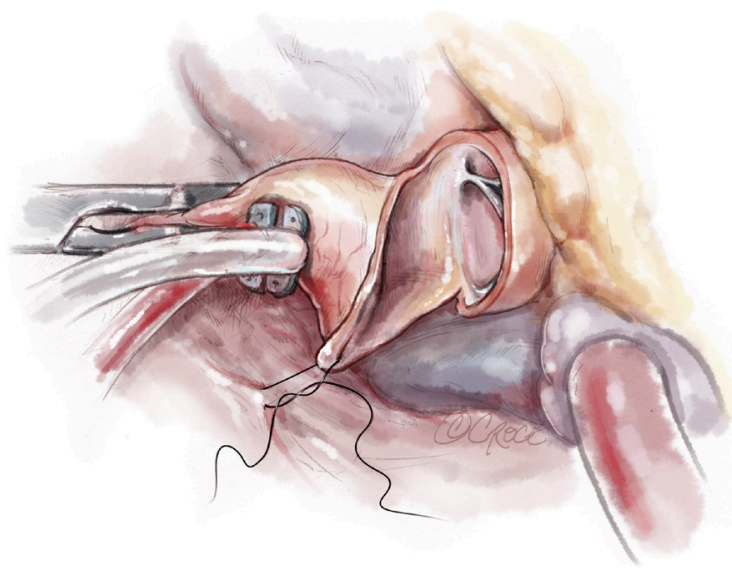

Figure 2 A transverse aortotomy is fashioned approximately $0.5 \mathrm{~cm}$ above the sinotubular junction. It is important not to cross the STJ with the incision as this prevents distortion of the aortic valve with closure of the aortotomy, which can result in aortic insufficiency. The distal aorta at the incision line is then tacked to the pericardial reflection at the level of the SVC for exposure.

elevates the aorta to the sternal level and facilitates visualization during the procedure. Cardiopulmonary bypass with single two-stage right atrial cannulation and venting of the left ventricle via the right superior pulmonary vein is established (Figure 1). The patient is systemically cooled to 34 degrees centigrade and following placement of an aortic cross clamp, normothermic cardioplegia solution is used to arrest the heart followed by cooling until the myocardium reaches 15 degrees centigrade. A transverse aortotomy is fashioned approximately $0.5 \mathrm{~cm}$ above the sinotubular junction (STJ). It is important not to cross the STJ with the incision as this may distort the aortic valve with closure of the aortotomy which can result in aortic insufficiency. The distal aorta at the incision line is then tacked to the pericardial reflection at the level of the superior vena cava for exposure (Figure 2).

\section{Surgical pearls}

\section{Operative technique}

A \#6 Ross retractor is used for exposure and is initially placed to distract the right coronary cusp to the aortic wall, with the inferior border of the retractor $2-3 \mathrm{~mm}$ above the cusp hinge point. This positioning provides excellent exposure of the sub-valvular outflow tract and protects the aortic valve leaflet. Using an 11 blade, the first incision is made $2.0 \mathrm{~mm}$ below the base of the right coronary leaflet insertion and $2.0 \mathrm{~mm}$ off the midline of the right coronary cusp towards the membranous septum Figure 3. The incision is made parallel to the LVOT (towards the apex) to a depth of the proposed resection, with the goal of a residual septal thickness of 0.8 to $1.0 \mathrm{~cm}$. A tendency is to direct the incision too far to the left of the apex. To avoid this, the surgeon can make the incision with the left hand. The length of the incision depends on the extent of the hypertrophy but is generally $35-50 \mathrm{~mm}$ long. In patients with concomitant mid-ventricular obstruction, the incision should extend to at least the level of the antero-septal papillary muscle head.

The second incision is approximately $2.0 \mathrm{~mm}$ in depth and $2.0 \mathrm{~mm}$ below the right coronary leaflet hinge and is carried into the sub-commissural area beginning at the base of the first incision. The third incision begins in the subcommissural area running parallel and $2.0 \mathrm{~mm}$ below the left coronary cusp hinge ending $4-5 \mathrm{~mm}$ from the mitral annulus (Figure 4).

Using Argentine forceps, the 'LVOT flap' is grasped and distracted inferiorly. Now a second $2.0 \mathrm{~mm}$ deep incision is made at the superior border of incisions 2 and 3 . In the region of the sub-commissural area the two original incisions are rounded with subsequent incisions. The process is 


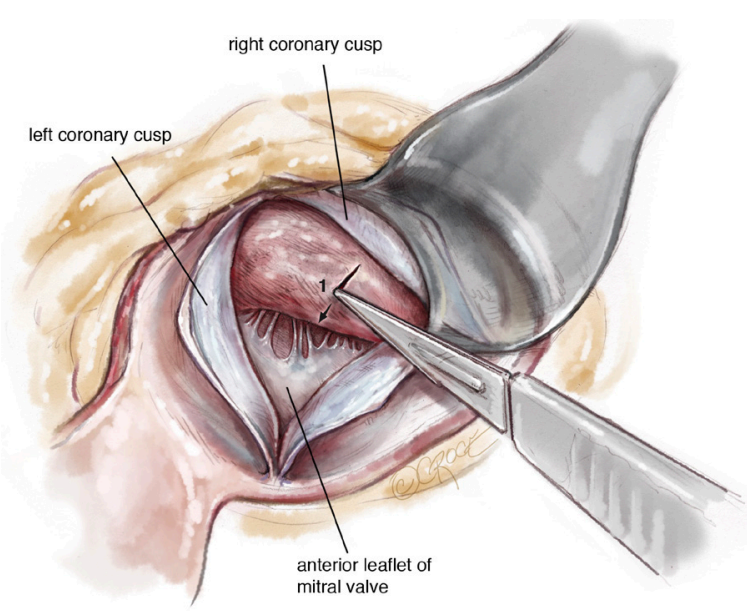

Figure 3 The first myectomy incision is made oriented towards the $\mathrm{LV}$ apex beginning $2 \mathrm{~mm}$ below the right coronary leaflet hinge point and $2 \mathrm{~mm}$ towards the membranous septum. The depth of the incision is usually $1-1.5 \mathrm{~cm}$ and length $3.5-5 \mathrm{~cm}$ as guided by the preoperative echocardiogram.

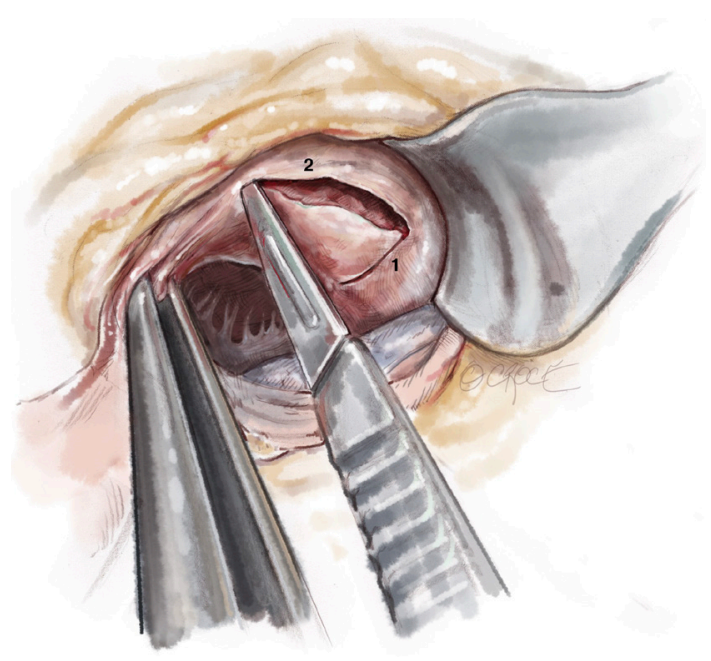

Figure 4 A series of $2.0 \mathrm{~mm}$ deep incisions running parallel to the LCC and RCC insertions are created to generate a myocardial flap corresponding to the desired resection thickness. Once the desired thickness has been generated, progress is made into the ventricle.

repeated eventually forming a flap in the LVOT septum with a desired resection thickness, usually $1.0-1.5 \mathrm{~cm}$. Now the Ross retractor can be repositioned across the valve into the newly formed defect. By distracting the specimen inferiorly, the resection can be advanced into the ventricle to achieve the desired length. By continuously assessing the

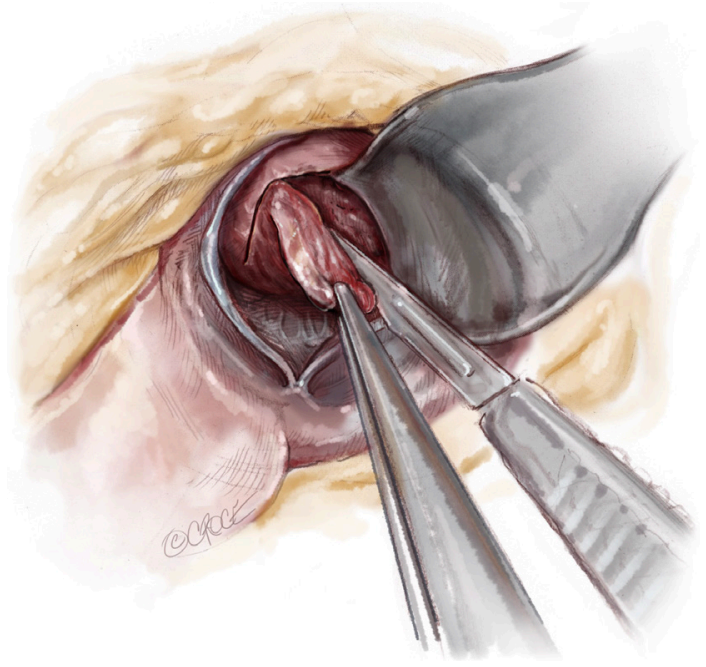

Figure 5 Using Argentine forceps, the 'LVOT flap' is grasped and distracted inferiorly. Now a second $2.0 \mathrm{~mm}$ deep incision is made at the superior border of incisions 2 and 3. In the region of the sub-commissural area the two original incisions are rounded with subsequent incisions. The process is repeated eventually forming a flap in the LVOT resection is terminated by placing downward traction on the specimen and incising across the base.

resection thickness looking on either side of the specimen as it is resected, constant thickness can be maintained. The lateral extent of the resection is $4-5 \mathrm{~mm}$ lateral to the mitral annulus. Resection is carried into the ventricle a distance determined by the preoperative TEE. Typical resections are between $35-45 \mathrm{~mm}$ in length. The resection is terminated by placing downward traction on the specimen and incising across the base. Care must be taken not to inadvertently cut the papillary muscles or chordae (Figure 5).

Not uncommonly, myocardial muscle bundles arise from the base of the specimen and can be divided on traction. The lateral aspect of the resection at the mitral annulus is completed with scissors, again with care to avoid trauma to the mitral sub-valvular apparatus Figure 6. Now the specimen should be easily delivered through the aortic annulus. The specimen can be measured at this point for adequacy. There is typically an area of scarring in the outflow tract which corresponds to repeated trauma caused by septal contact by systolic anterior motion (SAM). At a minimum, the resection should be $1.0 \mathrm{~cm}$ beyond the SAM septal contact point. Furthermore, the area of residual septum can be palpated. The thickness can be estimated by digital palpation and the transition to the distal ventricle assessed. Following resection, the base of the papillary 


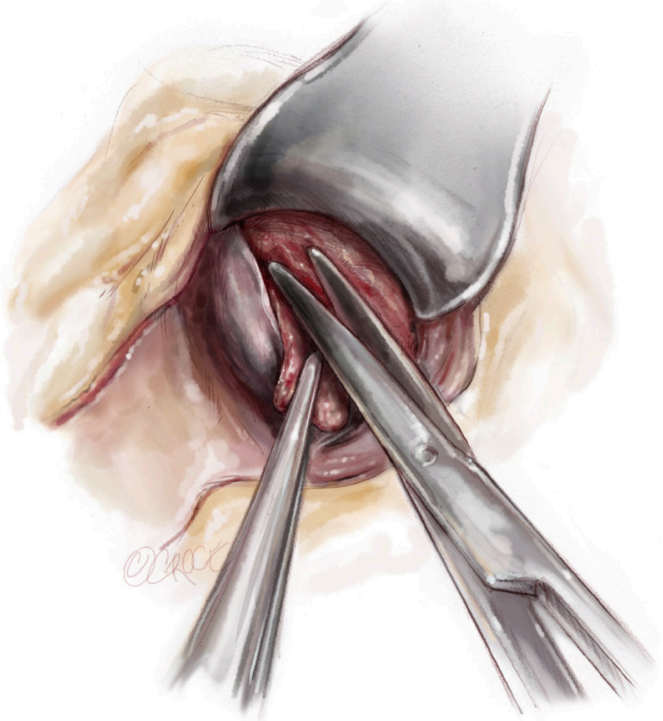

Figure 6 Final distal division made with scissors.

muscles and left ventricular apex should be easily visible through the outflow tract.

\section{Extension of resection}

With the retractor placed in the resection site, the distal base of the resection can be extended 1-2 cm laterally under the membranous septum with the resection narrowed as it is carried towards the annulus. By producing a traction point, the distal most portion of the resection can also be elongated if necessary (Figures 7,8 ).

Once complete, the resected area is swabbed with dental pledgets; free and tenuously attached muscle fragments can be identified in this way and removed. Finally, the left ventricular vent is clamped and the ventricular cavity is irrigated with saline solution and evacuated. The resection can usually be completed in 20 minutes with administration of only a single dose of cardioplegia solution. The aortotomy is most often closed in a single layer with a running monofilament suture (Figure 9,10).

Due to the infrequency of patients requiring septal myectomy, this operation is difficult to learn and master. Careful attention to preoperative, operative and postoperative details will ensure consistent results and minimize adverse outcomes.

\section{Intraoperative considerations}

Critical steps in the operation include adequate suspension
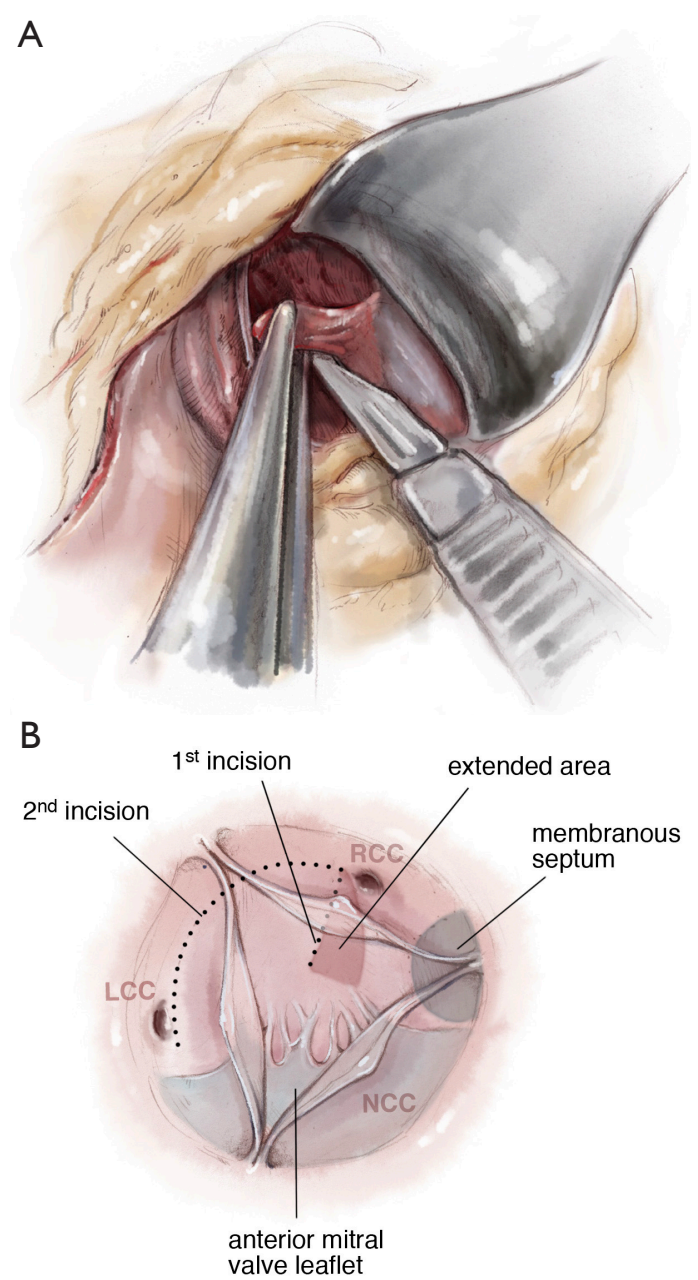

Figure 7 Extension of the resection under the membranous septum.

of the pericardium to elevate the aorta as much as possible in order to facilitate optimal visualization into the left ventricular cavity. Frequently, patients have cephalad displacement of the coronary ostia and it is critical to identify the right coronary artery prior to aortotomy to avoid inadvertent trauma. When performing the myectomy, the first incision must be parallel to the outflow tract directed towards the left ventricular apex or the resection will result in an LVOT 'corkscrew' configuration which will fail to relieve obstruction.

In addition, displacement of this incision towards the membranous septum increases the likelihood of postoperative heart block. If this incision is started further to the right, the bundle of His may be damaged. Following surgery, patients commonly develop left bundle branch 
block. The need for postoperative pacing following surgery in our experience is $4-6 \%$. Patients with pre-existing right bundle branch block should be cautioned that the need for postoperative pacing is a near certainty.

With incisions 2 and 3, it is important to generate the desired thickness of resection prior to advancing the resection into the left ventricular cavity. As one moves towards the apex, there is a progressively diminishing degree of freedom with respect to possible changes in the direction

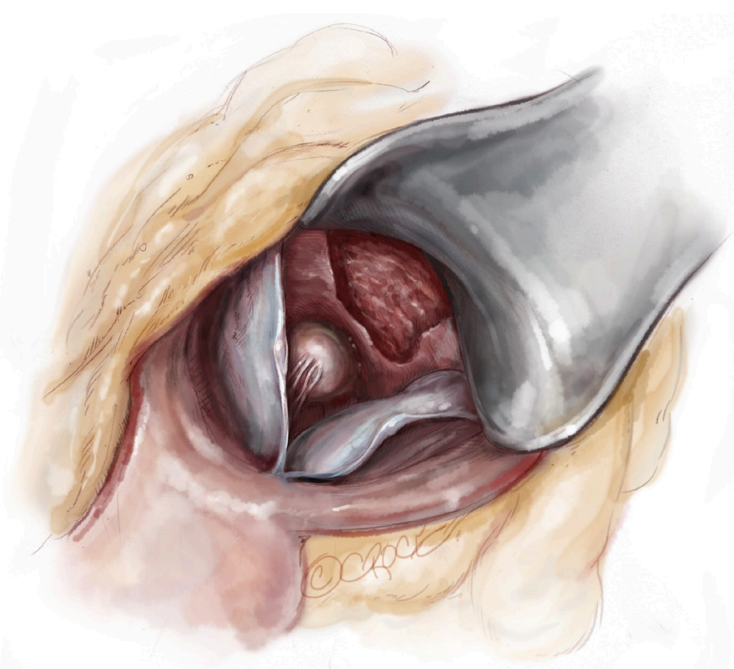

Figure 8 Completed resection. Note the papillary muscle heads are easily visible at the base of the resection.
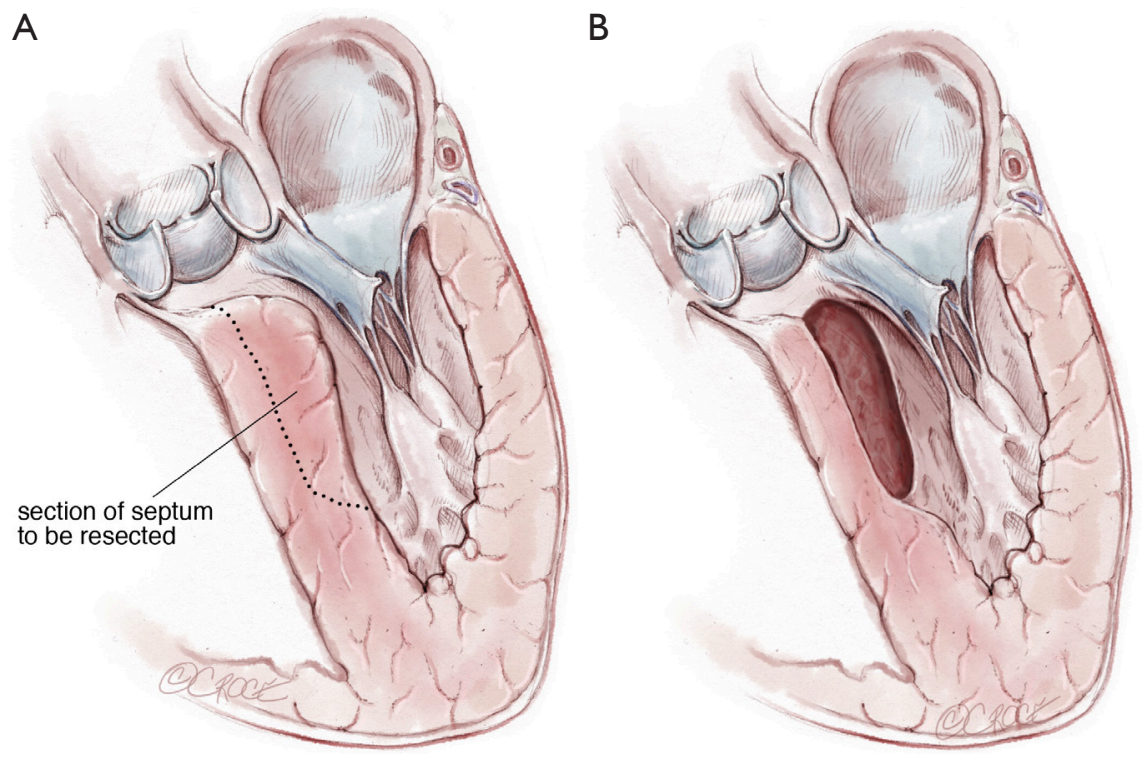

Figure 9 Illustration of septum resected.

and thickness of resection. The temptation to prematurely terminate the extent of resection should be avoided as traction on the specimen allows the resection to be carried further into the ventricle. Adequacy of the resection should be repeatedly evaluated with digital palpation as one advances. One should also monitor the thickness of resection by continuously visualizing the specimen resected, which is especially important as one rounds the specimen under the commissure between the right and left coronary cusps. In this region, the tendency is to flare the resection resulting in excessive thinning of the septum in this region. At its extreme, this will result in a ventricular septal defect (VSD) located in the right ventricular outflow tract. An adequate resection allows visualization of the base of the papillary muscles and usually extends to at least the level of the papillary muscle heads. Following resection, the thickness of the residual LVOT septum can be estimated via digital palpation. The septum should be between 0.8 and $1.0 \mathrm{~cm}$ thick and the transition into the trabeculated left ventricular cavity imperceptible.

Intraoperative TEE following weaning from bypass can provide information regarding the success of the resection. Careful inspection for a VSD is required. In our centre, a second pump run is warranted if there is significant residual turbulence either in the subvalvular or mid-cavitary regions or in the presence of a VSD. Resting gradients and gradients following premature ventricular contractions greater than $20 \mathrm{mmHg}$ require revision, where possible. 


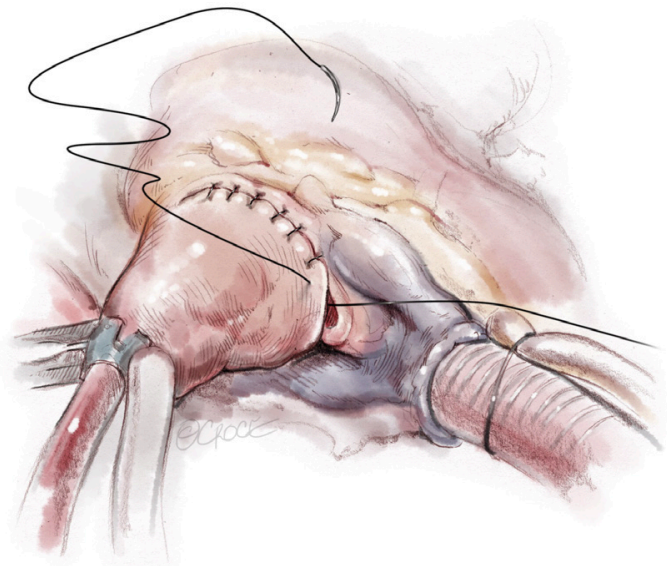

Figure 10 Aortic closure with 5-0 Prolene single layer suture.

Iatrogenic VSDs are fixed using a bovine pericardial patch. It is important to assess the aortic valve and ensure there is no new aortic regurgitation. While rare, this can result from excessive retraction during the resection. Importantly, the presence of mild central mitral regurgitation may be present on the TEE; however in our experience, this typically resolves postoperatively and likely represents transient ventricular injury following myectomy. Not infrequently, septal perforator flow may be seen in the region of resection on the postoperative TEE, which is characterized by flow directed into the left ventricular cavity during diastole.

\section{Completion}

Postoperative care follows standard considerations following cardiac surgery. Importantly, despite removal of the obstructive component of the septum, these patients can have significant diastolic dysfunction due to their underlying hypertrophic cardiomyopathy (HCM). These patients do well with maintenance of atrial-ventricular synchrony, avoidance of inotropes and afterload-reducing agents and maintenance of adequate preload. In addition, we routinely use aspirin and beta-blockers for the prevention of atrial fibrillation.

In addition, given the risk of injuring the bundle of His during the operation, patients with pre-existing RBBB will have a low threshold for insertion of a pacemaker and this is typically performed by postoperative day 4 . Patients extensive fibrosis detected on MRI, symptoms of syncope or documented ventricular tachycardia or fibrillation may be considered for insertion of an automatic implantable cardioverter-defibrillator prior to discharge.

\section{Conclusions}

Myectomy is the gold standard for treatment of LVOT obstruction. Excellent results can be obtained at centres with dedicated HCM programs. Careful attention throughout the preoperative, intraoperative and postoperative settings can ensure low complication rates and favourable long-term results.

\section{Acknowledgements}

We thank Ms. Beth Croce for drawing medical illustrations for our manuscript.

\section{Footnote}

Conflicts of Interest: The authors have no conflicts of interest to declare.

\section{References}

1. American College of Cardiology Foundation/American Heart Association Task Force on Practice, American Association for Thoracic Surgery, American Society of Echocardiography, et al. 2011 ACCF/AHA guideline for the diagnosis and treatment of hypertrophic cardiomyopathy: a report of the American College of Cardiology Foundation/American Heart Association Task Force on Practice Guidelines. J Thorac Cardiovasc Surg 2011;142:e153-203.

2. Maron BJ, Epstein SE, Morrow AG. Symptomatic status and prognosis of patients after operation for hypertrophic obstructive cardiomyopathy: efficacy of ventricular septal myotomy and myectomy. Eur Heart J 1983;4 Suppl F:175-85.

3. ten Berg JM, Suttorp MJ, Knaepen PJ, et al. Hypertrophic obstructive cardiomyopathy. Initial results and long-term follow-up after Morrow septal myectomy. Circulation 1994;90:1781-5.

4. Parry DJ, Raskin RE, Poynter JA, et al. Short and medium term outcomes of surgery for patients with hypertrophic obstructive cardiomyopathy. Ann Thorac Surg 2015;99:1213-9.

Cite this article as: Ralph-Edwards A, Vanderlaan RD, Bajona P. Transaortic septal myectomy: techniques and pitfalls. Ann Cardiothorac Surg 2017;6(4):410-415. doi: 10.21037/ acs.2017.07.08 